\title{
Characterization of biodegradable polymer blends of acetylated and hydroxypropylated sago starch and natural rubber.
}

\begin{abstract}
Development of biodegradable polymers from absolute environmental friendly materials has attracted increasing research interest due to public awareness of waste disposal problems caused by low degradable conventional plastics. In this study, the potential of incorporating natural rubber latex (NRL) into chemically modified sago starch for the making biodegradable polymer blends was assessed. Native sago starch was acetylated and hydroxypropylated before gelatinization in preparing starch thermoplastic using glycerol. They were than casted with NRL into biopolymer films according to the ratios of $100.00 / 0.00$, $99.75 / 1.25,98.50 / 2.50,95.00 / 5.00,90.00 / 10.00$ and $80.00 / 20.00 \mathrm{wt} / \mathrm{wt}$, via solution spreading technique. Water absorption, thermal, mechanical, morphological and biodegradable properties of the product films were evaluated by differential scanning calorimetry (DSC), universal testing machine (UTM), scanning electron microscopy (SEM) and fourier transform infrared spectroscopy. Results showed that acetylation promoted the incorporating behavior of NRL in sago starch by demonstrating a good adhesion characteristic and giving a uniform, homogenous micro-structured surface under SEM observation. However, the thin biopolymer films did not exhibit any remarkable trend in their DSC thermal profile and UTM mechanical properties. The occurrence of NRL suppressed water adsorption capacity and delayed the biodegradability of the biopolymer films in the natural environment. Despite the depletion in water adsorption capacity, all of the product films degraded $50 \%$ within 12 weeks. This study concluded that biopolymers with desirable properties could be formulated by choosing an appropriate casting ratio of the sago starch to NRL with suitable chemical substitution modes.
\end{abstract}

Keyword: Acetylation; Biodegradable polymer; Hydroxypropylation; Natural rubber; Sago starch. 